\title{
Fatores antropométricos associados à hipertensão arterial infantil ${ }^{*}$
}

\author{
Antropometric factors associated with \\ arterial hypertension in childhood
}

Hetty Nunes Lobo ${ }^{1}$ Renata Aparecida Elias Dantas ${ }^{2}$ Márcio Rabelo Mota ${ }^{3}$
Recebido em: 17/09/2016. Aprovado em: 19/01/2017.

1 Professora Mestre - Centro Universitário de Brasília - UniCEUB - Brasília.

2 Professora Doutora - Centro Universitário de Brasília - UniCEUB - Brasília.

3 Professor Doutor - Centro Universitário de Brasília - UniCEUB - Brasília.

\section{Resumo}

O presente estudo teve por objetivo verificar a associação entre a pressão arterial e o nível de atividade física em escolares da rede pública e privada do Distrito Federal. A amostra foi constituída por 212 estudantes (98 pública e 114 privada), na faixa etária de 7 a 10 anos. Os sujeitos foram submetidos a uma avaliação antropométrica, classificados de acordo com o IMC/idade e a um questionário de estilo de vida. A hipertensão foi definida quando a pressão arterial sistólica e/ou diastólica estavam acima do percentil 95 de acordo com a idade, sexo e estatura. Não foram evidenciados diferenças nas variáveis quando comparadas entre os quartis para o NAF. No entanto, quando comparadas entre escola pública e particular e entre pares, as crianças da escola privada apresentaram maior IMC e PA elevada. As crianças da escola privada apresentaram maiores fatores de risco cardiovascular do que as crianças da rede pública. Palavras-chave: Obesidade. Nível de Atividade Física. Crianças.

\begin{abstract}
This study aimed to investigate the association between blood pressure and physical activity in students from public and private schools in the Brazilian Federal District. The sample consisted of 212 students (98 public and 114 private), aged 7-10 years. The subjects underwent an anthropometric assessment, classified according to BMI / age and a lifestyle questionnaire. The of hypertension was defined as systolic blood pressure and / or diastolic blood pressure were above the 95th percentile according to age, sex and height. There were evident differences in variables between quartiles compared to the NAF. However when compared between public and private schools and between pairs of private school children had higher BMI and elevated BP. The private school children had higher cardiovascular risk factors than children from public schools.
\end{abstract}

Keywords: Obesity. Physical Activity Level. Children. 


\section{Introdução}

Ao final do século passado, uma grande parte dos estudos sobre nutrição, realizados no Brasil, concentrava-se nos aspectos da desnutrição. Contudo, dados recentes mostram diminuição no quadro de desnutrição e aumento de sobrepeso e obesidade em crianças, sendo esse fenômeno denominado "transição epidemiológico nutricional" que se caracteriza pela diminuição de doenças transmissíveis, porém, com concomitante aumento das doenças crônicas não transmissíveis (CINTRA et al., 2007; MCARDLE; KATCH; KATCH; 2010; PARIZZI et al., 2008).

No entanto, o excesso de peso constitui, atualmente, um grave problema de Saúde Pública, devido, principalmente, às elevadas taxas de prevalência, não somente em adultos, mas especialmente em crianças e adolescentes brasileiros (IBGE, 2010; LAMOUNIER et al., 2006). Como consequência, doenças anteriormente típicas de adultos (aterosclerose e hipertensão arterial), hoje, são processos iniciados na infância e estão, intimamente, relacionados ao excesso de adiposidade corporal (KUMANYIKA et al., 2008; DUMITHI et al., 2008; SAVITHA et al., 2007).

Entretanto, a abordagem preventiva associada a um estímulo à prática regular de atividade física e adoção de hábitos alimentares saudáveis, iniciado na infância e adolescência, pode ser uma boa estratégia, pois, nesse período, ocorre a formação do hábito (PARIZZI et al., 2008; LAMOUNIER et al., 2006). Acredita-se que um quadro de sobrepeso ou obesidade já instalado pode ser alterado mais facilmente nessa fase da vida, pois os hábitos alimentares e de atividade física ainda não estão completamente consolidados. Dessa maneira, o diagnóstico precoce do excesso de peso, PA elevada e sedentarismo, pode permitir o desenvolvimento de programas que estimulem a adoção de hábitos de vida saudáveis, que, por sua vez, possibilitariam o desenvolvimento salutar desses indivíduos. Além disso, poderá evitar que complicações de ordem fisiológicas e psicossociais, provocadas pela obesidade, interfiram na qualidade de vida do indivíduo, reduzindo, assim, as possibilidades desses indivíduos tornarem-se os adultos obesos de amanhã.

Dessa forma, o presente estudo teve o objetivo de verificar a prevalência entre a pressão arterial, IMC e o nível de atividade física (NAF) em escolas públicas e privadas de Brasília.

\section{Materiais e Métodos}

Este estudo se caracteriza como um estudo transversal de base populacional e foi encaminhado para Comitê de Ética em Pesquisa da Universidade Católica de Brasília- DF, aprovado no processo $n^{\circ} 227 / 11$.

A amostra foi obtida de forma randomizada e a partir de cálculo amostral com intervalo de confiança (IC) de 95\% para as escolas públicas e privadas em Brasília (DF). As escolas e turmas foram escolhidas aleatoriamente, preservando-se a proporcionalidade dos alunos matriculados em cada segmento de ensino. Foram analisados 212 estudantes (98 pública e 114 privada), sendo esse o número de alunos necessário para compor uma amostra representativa da população $(\mathrm{p}=0,05)$.

Após aprovação da Secretaria de Educação de Brasília e dos diretores das duas escolas, foi entregue aos alunos das escolas com idade entre 7 e 10 anos o termo de consentimento livre e esclarecido (TCLE) para ser assinado pelos pais. Aqueles que os pais permitiram a participação no estudo tiveram avaliação antropométrica, com peso, estatura, IMC e pressão arterial sistêmica. Os alunos escolhidos responderam um questionário de Barros e Nahas, (2003) para classificar o NAF. A aplicação desse questionário ocorreu em sala de aula, pelo próprio pesquisador.

\subsection{Medidas Antropométricas}

O índice de massa corporal (IMC) foi calculado considerando-se o quociente entre a massa corporal $\left(\right.$ Plenna $\left.^{\circ}\right)$ em quilogramas e a estatura em metros (estadiômetro SECA ${ }^{\oplus}$ 214, USA) quadrados $\left(\mathrm{kg} . \mathrm{m}^{-2}\right)$. De posse dos dados, todos os indivíduos foram classificados conforme sugerido pela World Health Organization (WHO,2006).

\subsection{Medidas Hemodinâmicas (pressão arterial e fre- quência cardíaca)}

As medidas de pressão arterial (Microlife BP 3AC1-1 com manguito para crianças) foram realizadas após os indivíduos permanecerem sentados por no mínimo cinco minutos em ambiente calmo e tranquilo. Além disso, os indivíduos deveriam estar sentados com pernas descruzadas, pés apoiados no chão, dorso recostado na cadeira e relaxado. Braços posicionados à altura do coração (nível do ponto médio do esterno ou quarto espaço intercostal), livre de roupas, apoiados com palma da mão voltada para cima e cotovelo ligeiramente fletido, confor- 
me procedimentos previamente descritos (NATIONAL, 2005).

A pressão arterial (PA) foi estratificada como elevada, quando os valores da pressão arterial sistólica (PAS) e/ou diastólica (PAD) foi igual ou superior ao percentil 95, ajustado a estatura, idade e sexo (NATIONAL, 2005).

A frequência cardíaca (FC) foi mensurada utilizando-se de um monitor de FC (Polar ${ }^{\varpi}$ S810i, Polar Electo Oy, Kempele, Finland) concomitantemente a medida de PA, bem como após aplicação do teste de avaliação da potência aeróbia proposto por Léger et al (1998) para confirmação do esforço máximo.

\subsection{Estimativa do nível de atividade física}

Para estimativa do nível de atividade física (NAF) semanal, os avaliados responderam um questionário de fácil compreensão, utilizando-se de figuras autoexplicativas que permitem levantar informações sobre: I. Aspectos demográficos (sexo e idade); II. Percepção de atividade física; III. Distância de casa até a escola; IV. Transporte utilizado para ir à escola; V. Atividades esportivas; VI. Tarefas domésticas e atividades de lazer do avaliado, por meio de informações de auto recordação. Nesse contexto foram considerados: a intensidade do esforço físico (leve, moderado e vigoroso) e a frequência semanal.

Para conversão das informações obtidas no questionário de atividade física em valores estimado de dispêndio energético, foi recorrido ao compêndio de atividades físicas que oferecem informações sobre o gasto energético em unidades do equivalente metabólico de trabalho (MET) (AINSWORTH et al., 2000).

Na sequência, foram estabelecidos pontos de cortes por meio do escore geral obtido na soma das atividades físicas, utilizando-se da classificação de quartis. Sendo consideradas, fisicamente, inativas as crianças com escore abaixo do percentil 25, insuficientemente ativas entre o percentil 25 e 50, moderadamente ativas entre o percentil 50 e 75 e ativas acima do percentil 75 .

\subsection{Tratamento Estatístico}

A normalidade dos dados foi testada por meio dos testes de Skewness e Kurtosis, tendo todas as variáveis apresentado valores entre $-1 \mathrm{e}+1$. Os dados estão expressos em média e $( \pm)$ desvio padrão bem como em frequência absoluta ( $\mathrm{n}$ ) e relativa (\%). O Power da amostra $(n=212)$ foi de $87 \%$ com um alfa de $5 \%$ para análise. $O$ teste $t$ de Student não pareado foi aplicado para comparar todas as variáveis investigadas (IMC, PAS, PAD e NAF) entre as escolas (pública e particular). Posteriormente, a amostra foi dividida em quartis, considerando a classificação do NAF entre os percentis 25, 50 e 75. Oneway ANOVA com post hoc de Bonferroni foi utilizado para comparar o IMC, PAS e PAD entre os quartis $\left(1^{\circ}, 2^{\circ}, 3^{\circ}\right.$ e $4^{\circ}$ ).

Ademais, ANOVA two way com post hoc de Bonferroni foi aplicada para comparar todas as variáveis (IMC, PAS, PAD e NAF) entre sexos (meninos e meninas), bem com entre escolas (pública e particular). Por fim, o teste de Qui-quadrado foi empregado para realizar comparações entre frequências (\%) para o IMC e PA. O nível de significância adotado foi de $5 \%(\mathrm{p}<0,05)$. Para tanto, foram utilizados os softwares SPSS 15.0 para Windows e GPower 3.0.10.

\section{Resultados}

A caracterização da amostra com os valores das médias e desvios padrão das variáveis antropométricas (massa corporal, estatura e IMC), hemodinâmicas (PAS, PAD e FC), bem como nível de atividade física, são apresentados na tabela 1 .

Tabela 1. Características descritivas da amostra $(n=212)$. Dados expressos em média e ( \pm ) desvio padrão.

\begin{tabular}{|c|c|}
\hline Variáveis & Média e $( \pm)$ desvio padrão \\
\hline Idade (anos) & $9,7 \pm 1,4$ \\
\hline Massa corporal (kg) & $31,7 \pm 6,5$ \\
\hline Estatura (cm) & $142,2 \pm 11,5$ \\
\hline IMC (kg.m-2) & $16,4 \pm 2,3$ \\
\hline $\operatorname{PAS}(\mathrm{mmHg})$ & $93,7 \pm 14,9$ \\
\hline PAD (mmHg) & $57,2 \pm 12,1$ \\
\hline NAF (MET.semana-1) & $554,5 \pm 275,1$ \\
\hline
\end{tabular}

Quando a amostra foi dívida em quartis para o nível de aptidão física, sendo o $1^{\circ}$ quartil o de mais baixo nível de atividade física e o $4^{\circ}$ quartil o nível mais alto, nenhuma das variáveis investigadas (IMC, PAS e $\mathrm{PAD})$ apresentaram diferenças significativas $(\mathrm{p}>0,05)$. A média para o IMC $\left(\mathrm{kg} \cdot \mathrm{m}^{-2}\right)$, para o $1^{\circ}, 2^{\circ}, 3^{\circ}$ e $4^{\circ}$ quartil, foi de $17,5 \pm 3,18,1 \pm 3,7,17,3 \pm 3,4,18,3 \pm 3,5$, já para a PAS (mmHg), os quartis apresentaram uma média de $94,0 \pm 13,9,96,3 \pm 15,8,90,1 \pm 13,5,93,9 \pm 16,1$ e para a PAD 
(mmHg) 57,5 $\pm 11,4,58,3 \pm 13,2,56,8 \pm 11,8,56,2 \pm 12,3$ nessa ordem consequentemente.

Por outro lado, quando comparado as variáveis investigadas entre estudantes da escola pública e privada, todas as variáveis (IMC, PAS e PAD), exceto idade e NAF, apresentaram diferenças significativas, sendo que, alunos da escola privada apresentaram maior IMC $\left(19,1 \pm 3,8\right.$ vs $\left.16,3 \pm 2,3 \mathrm{~kg} \cdot \mathrm{m}^{-2} ; \mathrm{p}=0,001\right)$, maiores valores de PAS $(99,2 \pm 17,1$ vs $87,3 \pm 8,0 \mathrm{mmHg} ; \mathrm{p}=0,001)$ e PAD $(60,5 \pm 14,4$ vs 53,4 $\pm 7,2$ mmHg; $\mathrm{p}=0,001)$ (Tabela 2).

Tabela 2. Comparação do IMC, PAS e PAD entre os estudantes da escola pública e privada. Dados expressos em média e $( \pm)$ desvio padrão.

\begin{tabular}{lccc}
\hline Variáveis & Pública $(\mathrm{n}=98)$ & Privada $(\mathrm{n}=114)$ & $\mathrm{p}$ \\
\hline Idade (anos) & $8,7 \pm 0,9$ & $10,3 \pm 1,3$ & 0,067 \\
NAF (MET.semana $\left.{ }^{-1}\right)$ & $560,9 \pm 283,9$ & $548,9 \pm 269,2$ & 0,910 \\
IMC (kg.m-2) & $16,3 \pm 2,3$ & $19,1 \pm 3,8$ & 0,001 \\
PAS (mmHg) & $87,3 \pm 8,0$ & $99,2 \pm 17,1$ & 0,001 \\
PAD (mmHg) & $53,4 \pm 7,2$ & $60,5 \pm 14,4$ & 0,001 \\
\hline
\end{tabular}

$\mathrm{IMC}=$ índice de massa corporal; $\mathrm{PAS}=$ pressão arterial sistólica; $\mathrm{PAD}=$ pressão arterial diastólica; NAF= nível de atividade física.

Quando realizadas comparações entre sexos e entre escolas, todas as variáveis, exceto pelo NAF ( $\mathrm{p}>0,05)$, foram maiores em estudantes de ambos os sexos da escola particular.

Meninos e meninas apresentaram frequência (\%) de IMC normal e excesso de peso semelhante $(\mathrm{p}=0,791)$. Ademais, também, não foram evidenciadas diferenças significativas de frequências entre os sexos quanto à estratificação da PA em normal e elevada $(p=0,215)$.

Tabela 3. Comparação do IMC, PAS e PAD entre os estudantes da escola pública e privada bem como entre gêneros. Dados expressos em média e $( \pm)$ desvio padrão.

\begin{tabular}{lcccc}
\hline & Escola Pública & \multicolumn{3}{c}{ Escola Privada } \\
\cline { 2 - 5 } Variáveis & $\begin{array}{c}\text { Meninos } \\
(\mathrm{n}=62)\end{array}$ & $\begin{array}{c}\text { Meninas } \\
(\mathrm{n}=36)\end{array}$ & $\begin{array}{c}\text { Meninos } \\
(\mathrm{n}=76)\end{array}$ & $\begin{array}{c}\text { Meninas } \\
(\mathrm{n}=38)\end{array}$ \\
\hline $\begin{array}{l}\text { NAF (MET. } \\
\left.\text { semana }{ }^{-1}\right)\end{array}$ & $586,0 \pm 312,0$ & $517,9 \pm 222,0$ & $546,7 \pm 267,1$ & $564,6 \pm 279,5$ \\
IMC (kg.m $\left.{ }^{-2}\right)$ & $16,2 \pm 2,4$ & $16,5 \pm 2,2$ & $19,1 \pm 3,9^{*}$ & $19,4 \pm 3,7^{*}$ \\
PAS (mmHg) & $86,6 \pm 8,2$ & $88,5 \pm 7,4$ & $98,4 \pm 17,1^{*}$ & $101,4 \pm 17,6^{*}$ \\
PAD (mmHg) & $53,1 \pm 7,5$ & $53,9 \pm 6,4$ & $60,0 \pm 14,2^{*}$ & $61,9 \pm 15,1^{* 4}$
\end{tabular}

* = diferença significativa entre sexos e entre as escolas $(p<0,05)$. IMC = índice de massa corporal; $\mathrm{PAS}=$ pressão arterial sistólica; $\mathrm{PAD}=$ pressão arterial diastólica; $\mathrm{NAF}=$ nível de atividade física.
No entanto, os estudantes da escola pública apresentam frequência de IMC e PA normal estatisticamente diferente de estudantes da rede privada. A frequência de estudantes da escola pública com IMC estratificado como excesso de peso foi de $20,4 \%$, ao passo que estudantes da rede privada apresentaram $53,5 \%$, sendo esses valores diferentes entre si $(p=0,001)$.

Do mesmo modo, quando verificada a PA, os estudantes das escolas privada e pública apresentaram frequências de PA normal e elevada estatisticamente diferentes $(\mathrm{p}=0,0001)$. Apenas $1 \%$ dos estudantes de escola pública apresentam PA elevada, ao passo que 38,6\% dos alunos de escola privada foram estratificados com valores de PA acima do recomendado.

Tabela 4. Distribuição (n), prevalência (\%) por escola e para a amostra total, quanto à classificação do índice de massa corporal (IMC) e pressão arterial (PA).

\begin{tabular}{|c|c|c|c|c|c|}
\hline & \multicolumn{2}{|c|}{ Escola Pública (n=98) } & \multicolumn{2}{|c|}{ Escola Privada ( $n=114$ ) } & \multirow[t]{2}{*}{$\mathrm{p}$} \\
\hline & $\mathrm{n}$ & $\%$ & $\mathrm{n}$ & $\%$ & \\
\hline \multicolumn{6}{|l|}{ IMC } \\
\hline Normal & 78 & 79,6 & 53 & 46,5 & \multirow{2}{*}{0,0001} \\
\hline Excesso de peso & 20 & 20,4 & 61 & 53,5 & \\
\hline \multicolumn{6}{|l|}{ PA } \\
\hline Normal & 97 & 99,0 & 70 & 61,4 & \multirow{2}{*}{0,0001} \\
\hline Elevada & 1 & 1,0 & 44 & 38,6 & \\
\hline
\end{tabular}

\section{Discussão}

Os resultados do presente estudo apontam que o NAF parece não afetar a adiposidade corporal (IMC) e PA no estrato de idade investigado (escolares de 7 a 10 anos), uma vez que não foram evidenciadas diferenças estatísticas quando comparado os quartis de NAF para as variáveis acima mencionadas. Da mesma forma, Rosa et al (2011), ao compararem o nível de atividade física habitual (NAFH) em crianças e adolescentes por meio do pedômetro, não demonstraram diferença estatística entre o IMC de meninos e meninas divididos pelo NAFH. Além disso, Parzianello e Santos (2007), também, não demonstram associações significantes entre o NAFH e adiposidade corporal em crianças da mesma faixa etária do presente estudo (7 a 10 anos).

Quando comparados os valores de PAS e PAD entre os quartis do NAF, também não foram identificadas diferenças estatísticas, o que, também, foi evidenciado no estudo de Hoffmann et al (2010) em que os autores não 
encontraram associação entre o NAF e classificação da PA em escolares de Caxias do Sul.

Um resultado interessante do presente estudo refere-se ao fato de que meninos e meninas da escola privada apresentam maiores valores de IMC, PAS e PAD, o que está em descordo com os achados de Silva, Lopes e Silva (2011) demonstraram que estudantes da rede privada de ensino apresentam menor prevalência de excesso de peso e PA elevada quando comparado a seus pares da rede pública de ensino.

Por outro lado, alguns estudos Ferreira et al. (2008) e Mendonça (2010) que investigaram estudantes da cidade de Taguatinga-DF e Maceió-AL, também apontam haver uma maior prevalência de excesso de peso em estudantes da escola particular quando comparado aos da rede pública, independentemente do sexo. Além disso, Mendonça et al (2010), ao avaliar estudantes da rede pública e privada de ensino da cidade de Maceió, reporta que estudantes da rede privada de ensino com idade entre 7 a 17 anos, apresentam 2,2 vezes mais chance de terem sobrepeso (Odds Ratio= 2,2; IC95\%: 1,36-3,32) e, aproximadamente, cinco vezes mais chances de apresentarem obesidade (Odds Ratio= 4,7; IC95\%: 2,32-9,34) em relação aos das escolas públicas.

Analisando a tabela 4, foram identificadas diferenças estatísticas entre o IMC e a PAS da escola pública e privada. Resultados semelhantes foram observados em estudos como o de Ribeiro e et al (2006), ao avaliarem 1.450 estudantes de 6 a 18 anos, mostraram que os indivíduos com IMC elevado apresentam 3,6 e 2,7 vezes mais risco de ter pressão arterial sistólica (PAS) e pressão arterial diastólica (PAD) elevadas, respectivamente. Para Monego e Jardim (2006), ao estudarem 3.169 escolares, observaram associação significante entre excesso de peso e PAS. No entanto, Guimarães et al. (2008), ao avaliarem dados de pressão arterial e IMC de 536 adolescentes de 11 a 18 anos, concluíram que o aumento percentual de PAS e PAD elevadas acompanhou a elevação do IMC e que cada aumento no IMC elevaria a PAS em 1,198 mmHg.

Ademais, Garcia et al. (2004), ao avaliarem 672 crianças entre 2 e 11 anos da cidade de Belo Horizonte - MG, demonstraram que estudantes da rede privada de ensino apresentam maiores valores de PAS $(106,3 \pm 10,2$ vs $100,2 \pm 11,7 \mathrm{mmHg} ; \mathrm{p}=0,026)$ e PAD $(64,4 \pm 12,1$ vs $57,8 \pm 13,5$ mmHg; $\mathrm{p}=0,03)$ do que estudantes da rede pública.
Adicionalmente, vale salientar que a prevalência de excesso de peso e PA elevada da escola particular se mostrou bem maior do que em estudos anteriormente realizados por Silva, Lopes e Silva (2011) e por Garcia et al. (2004), sugerindo que a escola particular investigada, desenvolva programas para estimular a adoção de hábitos de vida saudáveis, tais como: incentivar a prática regular de atividade física bem como programas de reeducação alimentar.

Dentre as limitações desse estudo, um fator importante a ser citado é que a não avaliação do estágio maturacional e das dobras cutâneas podem ter afetado os resultados. Sugere-se que estudos futuros analisem, também, a aptidão cardiorrespiratória controlando essas variáveis.

\section{Conclusão}

Conclui-se que as crianças da escola privada demonstram maiores fatores de risco cardiovascular do que crianças da escola púbica de Brasília, uma vez que apresentaram valores maiores de IMC, PAS e PAD, quando comparado a seus pares da rede pública de ensino, além de, também, apresentarem uma maior prevalência de excesso de peso e PA elevada.

Por fim, acreditando nos resultados encontrados no estudo, esperamos que venham surgir novos trabalhos e programas por parte do governo destinando o espaço maior aos professores de Educação Física para o desenvolvimento de atividades profiláticas, voltadas à conscientização dos benefícios de um estilo de vida ativo para as crianças a fim de que tenham, no futuro, uma vida mais saudável.

\section{Referências}

AINSWORTH, B. E. et al. Compendium of physical activities: an update of activity codes and met intensities. Medicina \& Science in Sports \& Exercise, Hagerstown, v. 32, n. 9(Suppl.), p. S498-S504, Sep. 2000.

BARROS, M. V. G; NAHAS, M. V. Medidas da atividade física: teoria e aplicação em diversos grupos populacionais. Londrina: Midiograf, 2003.

CINTRA, I. P. et al. Evolução de duas séries históricas do índice de massa corporal em adolescentes. Jornal de Pediatria, Rio de Janeiro, v. 83, n. 62. p. 157, maio 2007. doi: 10.1590/S0021-75572007000200010 
DUMITHI, S. C. et al. Aptidão física relacionada à saúde de alunos do ensino fundamental do município de Rio Grande. Revista Brasileira de Medicina e Esporte, Niterói, v. 14, n. 5, p. 152-160, 2008. doi: 10.1590/S151786922008000500011

EIBERG, S. H. H. Maximum oxygen uptake and objectively measured physical activity in Danish children 6-7 years of age: the Copenhagen school child intervention study. British Journal of Sports Medicine, Oslo v. 39, n. 10, p. 725-30, Oct. 2005. doi: 10.1136/bjsm.2004.015230

FERREIRA, A. P. et al. Prevalência de sobrepeso e obesidade em escolares de Taguatinga - DF. Revista do Instituto de Ciência da Saúde, Brasília, v. 26, n. 2, p.161-166, abr./jun. 2008.

GARCIA, F. D. et al. Avaliação de fatores de risco associados com elevação da pressão arterial em crianças. Jornal Pediatria, Rio de Janeiro, v. 80, n. 1, p. 29-34, jan./fev. 2004. doi: 10.2223/JPED.1130

GUIMARÃES, I. C. B. et al. Pressão arterial: efeito do índice de massa corporal e da circunferência abdominal em adolescentes. Arquivo Brasileiro de Cardiologia, São Paulo, v. 90, n. 6, p. 293-299, jun. 2008. doi: 10.1590/ S0066-782X2008000600007

HOFFMANN, M.; SILVA, A. C. P.; SIVIERO, J. Prevalência de hipertensão arterial sistêmica e interrelações com sobrepeso, obesidade, consumo alimentar e atividade física, em estudantes de escolas municipais de Caxias do Sul. Pediatria, São Paulo, v. 32, n. 3, p.163-72, jul./set. 2010.

INSTITUTO BRASILEIRO DE GEOGRAFIA E ESTATÍSTICA. Pesquisa de Orçamentos Familiares: 20092010. Disponível em: <www.ibge.gov.br>. Acesso em: 15 set. 2011.

KUMANYIKA, S. K. et al. Population-based prevention of obesity: the need for comprehensive promotion of healthful eating, physical activity, and energy balance. A scientific statement from American heart association council on epidemiology and prevention, interdisciplinary committee for prevention (formerly the expert panel on population and prevention science). Circulation, Hagerstown, v. 118, p. 428-64, jul. 2008. doi: 10.1161/ CIRCULATIONAHA.108.189702

LAMOUNIER, J. A. et al. Obesidade e dislipidemias. Programa Nacional de Educação Continuada em Pediatria da Sociedade Brasileira de Pediatria, PRONAP, Brasíia, v. 6, n. 4, p. 23-70, dez. 2006.

LÉGER, L. A. et al. The multistage 20-metre shuttle run test for aerobic fitness. Journal of Sports Sciences, London, v. 6, n. 2, p. 93-101, jun. 1988. doi: $\underline{10.1080 / 02640418808729800}$
MCARDLE, W. D.; KATCH, F. I.; KATCH, V. L. Fisiologia do exercício. 7. ed. Rio de Janeiro: Guanabara Koogan, 2010.

MENDONÇA, M. R. T. et al. A prevalência de sobrepeso e obesidade em crianças e adolescentes da cidade de Maceió. Revista da Associação Médica Brasileira, São Paulo, v. 56, n. 2, p. 192-196, 2010. doi: 10.1590/S010442302010000200018

MONEGO, E. T.; JARDIM, P. C. B. V. Determinantes de risco para doenças cardiovasculares em escolares. Arquivo Brasileiro de Cardiologia, São Paulo, v. 87, n. 1, p. 37 45, jul. 2006. doi: 10.1590/S0066-782X2006001400006.

NATIONAL High Blood Pressure Program Working Group on Hypertension Control in Children and Adolescents. The fourth report on the diagnosis, evaluation, and treatment of high blood pressure in children and adolescents. Pediatrics, Springfield, v. 114, Suppl. 2, p. 555-576, Aug. 2005.

PARIZZI, M. R. et al. Abordagem interdisciplinar do adolescente obeso com ênfase nos aspectos psicossociais e nutricionais. Revista Médica de Minas Gerais, Belo Horizonte, v. 18, n. 4 (Suppl. 1), p. 154-160, nov. 2008.

PARZIANELLO, R. P.; SANTOS, M. A. M. Correlação entre o índice de massa corporal e o nível de atividade física habitual em crianças de 7 a 10 anos. Revista Brasileira de Prescrição de Fisiologia do Exercício, São Luis, v. 1, n. 1, p. 45-54, jan./fev. 2007.

RIBEIRO, R. Q. C. et al. Fatores adicionais de risco cardiovascular associados ao excesso de peso em crianças e adolescentes. O estudo do coração de Belo Horizonte. Arquivo Brasileiro de Cardiologia, São Paulo, v. 86, n. 6, p. 408418, jun. 2006. doi: 10.1590/S0066-782X2006000600002

ROSA, C. S. C. et al. Atividade física habitual de crianças e adolescentes mensurada por pedômetro e sua relação com índices nutricionais. Revista Brasileira Cineantropometria e Desempenho Humano, Florianópolis, v. 13, n. 1, p. 22 28, jan./fev. 2011. doi: 10.5007/1980-0037.2011v13n1p22.

SAVITHA, M. R. et al. Essential hypertension in early and mid-adolescence. Indian Journal Pediatrics, New Delhi, v. 74, n. 11, p. 1007-1011, nov. 2007.

SILVA, K. S.; LOPES, A. S; SILVA, F. M. Atividade física no deslocamento à escola e no tempo livre em crianças e adolescentes da cidade de João Pessoa, PB, Brasil. Revista Brasileira de Ciência e Movimento, Brasília, v. 15, n. 3, p. 61-70, jul./set. 2011. doi: 10.18511/rbcm.v15i3.761

WORLD HEALTH ORGANIZATION. Child Growth Standards: Length/height-for-age, weight-for-age, weight-for-length, weight-for-height and body mass index-for-age. Methods and development. WHO (nonserial publication). Geneva, Switzerland: WHO, 2006. 